Cinémas

Revue d'études cinématographiques

Revue d'études cinématographiques

Journal of Film Studies

\title{
Présentation
}

\section{Elena Dagrada}

Volume 13, numéro 1-2, automne 2002

Limite(s) du montage

URI : https://id.erudit.org/iderudit/007953ar

DOI : https://doi.org/10.7202/007953ar

Aller au sommaire du numéro

Éditeur(s)

Cinémas

ISSN

1181-6945 (imprimé)

1705-6500 (numérique)

Découvrir la revue

Citer ce document

Dagrada, E. (2002). Présentation. Cinémas / Revue d'études cinématographiques, 13(1-2), 7-9. https://doi.org/10.7202/007953ar d'utilisation que vous pouvez consulter en ligne.

https://apropos.erudit.org/fr/usagers/politique-dutilisation/ 


\section{Présentation}

\section{Elena Dagrada}

En dépit de sa vocation synchronique et universelle, toute théorie du montage est nécessairement ancrée dans l'histoire. Ce n'est peut-être pas un hasard si les périodes principales de réflexion sur le montage, dont nous sommes les héritiers, ont aussi été des moments de réflexion sur le langage et/ou de combat idéologique qui, semble-t-il, ont laissé des traces dans notre conception même du montage jusqu'à aujourd'hui.

Il n'est pas question ici de reprendre en d'autres mots la problématique du montage telle qu'elle a été envisagée (sinon épuisée) au cours des dernières années - à savoir en fonction de sa capacité narrative ou langagière d'une part, et de ses rapports à l'idéologie de l'autre: deux options que la sémiologie, la narratologie et la critique militante ont privilégiées avec des résultats fort convaincants. Comme le titre même de ce numéro le suggère, il s'agit ici de réfléchir sur les frontières du montage, entendues dans un double sens: d'abord, comme seuils de la légitimité du recours au montage durant certaines périodes historiques, comme celle de la modernité cinématographique, alors que son idée est inévitablement associée à la notion de faux, de mensonge ou de truc, en raison de genres bien entendu qui se veulent "authentiques", comme le documentaire; ensuite, comme cas limites de montage, tels le cinéma-vérité, le cinéma de la continuité ou le cinéma des premiers temps, qui par le refus, l'absence ou la dissimulation du montage, souvent peu évident à première vue, nous obligent à revoir cette notion, de par le doute qu'ils font peser sur ses limites. Enfin, pour terminer par la bonne bouche, il y a lieu de porter la réflexion sur ces autres cas limites que sont l'image numérique, l'édition par ordinateur, le cinéma expérimental, le cinéma d'animation. 
Lobjectif de ce numéro n'est donc pas de revenir sur la définition du montage ni de faire son historique, mais plutôt de mettre en cause ses taxinomies plus traditionnelles, et ce à cause de l'impasse actuelle où elles se trouvent. En effet, les pratiques cinématographiques liées aux mutations technologiques - ni plus ni moins que les découvertes historiques concernant le cinéma des premiers temps ou la relecture du modèle classique - exigent que nous réfléchissions en vue d'une re-définition du montage qui tiendrait compte, entre autres, de différents domaines jusqu'ici quelque peu négligés et qui privilégierait, notamment, une approche qui soit consciente de l'intersection du montage filmique avec d'autres pratiques artistiques.

Ainsi, les articles ici recueillis abordent le montage suivant une approche nouvelle. De plus, ils s'efforcent de reconsidérer cette question du montage à partir de ses champs d'application, notamment selon l'épistémé de la fin du XIX'-début du XXe siècle, afin de cerner quelques-unes des notions à l'œuvre dans sa dimension opérative, à savoir la mécanicité, la discontinuité, la superposition (Albera). Ils mettent en évidence le paradoxe qui habite le passage de la singularité du photogramme à la multiplicité de sa mise en série dans l'image filmique (Gaudreault). Ils questionnent sa pratique dans la production filmique du cinéma allemand des années 1920, parallèlement à l'absence de sa conceptualisation (Kessler). Ils passent en revue les modalités du montage au sein des nouvelles technologies numériques (Lefebvre et Furstenau) et du cinéma expérimental, afin d'y repérer les formes contemporaines de la pratique du remploi, voire une constante de l'histoire de l'art, que le cinéma ne cesse d'enrichir au fur et à mesure (Brenez). Ils analysent enfin le fonctionnement de certaines figures de montage comme le two-shot et le champ-contrechamp dans la représentation visuelle des dialogues (Baetens), ainsi que les véritables limites d'intervention du montage dans le cinéma d'animation (Tomasovic) ou dans un certain cinéma d'auteur, imperméable à toute sorte d'imposition apocryphe de re-montage (Dagrada).

Mais les études réunies dans ce numéro poursuivent également le questionnement sur le montage par l'analyse de quelques cas particuliers: celui du photographe Gustave Le Gray qui 
en 1895 inaugure des "montages photographiques" et de Georges Méliès chez qui le montage "joue de la référence au corps mécanique, dissociable, superposable»(Albera); ceux de Niépce, de Marey, de Muybridge, de Lumière même et de ses vues à la fois singulières et multiples (Gaudreault); la pratique $\mathrm{du}$ found footage dans ses variations élégiaque, critique, structurelle, matériologique, analytique (Brenez); celle du dialogue filmé comme modèle réduit du film dans deux cas de comédie hollywoodienne des années 1930 - It Happened One Night, Bringing Up Baby - confrontées à deux titres plus récents Orlando, The Color Purple (Baetens); la pertinence des théorisations baziniennes, eisensteiniennes et peirciennes en ce qui a trait au devenir du cinéma sous l'effet du numérique (Lefebvre et Furstenau); trois films allemands - Le Docteur Mabuse, Nosferatu, Metropolis - où la présence inattendue d'effets de montage rend précaire le statut de l'image (Kessler); les variations du montage dans les versions américaine et italienne de Stromboli de Roberto Rossellini (Dagrada) et ce cas limite par excellence que sont les films hybrides conjuguant la prise de vues « réelles» avec l'animation (Tomasovic).

L'enjeu, somme toute, est nul autre que celui de questionner ces cas limites afin qu'ils nous permettent de mieux discerner les limites mêmes du montage, sans oublier les limites, nécessairement ancrées dans l'histoire, qui ont guidé toute réflexion théorique sur le montage jusqu'à aujourd'hui.

Università degli Studi di Milano 